\title{
Electronic Structure Calculations of InP-Based Coupled Quantum Dot-Quantum Well Structures
}

\author{
J. ANDRZEJEWSKi
}

Laboratory for Optical Spectroscopy of Nanostructures,

Department of Experimental Physics, Faculty of Fundamental Problems of Technology,

Wrocław University of Technology, Wybrzeże Wyspiańskiego 27, 50-370 Wrocław, Poland

In this work we investigate the electronic structure of coupled $0 \mathrm{D}-2 \mathrm{D}$ nanostructures. The respective confined state energy levels in a quantum dot-quantum well system are calculated for various conduction band offsets between the quantum dot and surrounding material. The calculated electron and hole energy levels with their wave functions allow determining if the wave functions are within the injector quantum well or within the quantum dot and if the carrier positions on the energy scale are appropriate from the point of view of a possible laser structure utilizing the so-called tunnel injection scheme. It is shown that for an adequate width of an injector quantum well and the conduction band offsets designing an optimal tunnel injection structure is possible.

DOI: 10.12693/APhysPolA.129.A-97

PACS: 73.21.La, 73.21.Fg, 73.22.Dj, 74.50.+r

\section{Introduction}

Semiconductor quantum dots (QD) are promising as an active material in laser devices, because QD-based lasers have weaker temperature-sensitivity of the threshold current, as compared to quantum well (QW) based lasers [1]. However, a real QD laser presents several problems that need to be overcome, such as temperature sensitivity of the threshold current density due to the parasitic recombination outside of QDs [2] or sublinearity of light-current characteristics [3]. There are also other issues in actual QD structures, associated with the random size, shape, and density of QDs, and the limitations of carrier collection and their redistribution among the QDs. In an attempt to deal with these problems, the QD laser has recently been modified into a QD-QW laser via tunnel-injection of electrons from the QW into the QDs, which was proposed in Refs. [4] and [5]. Such a tunnel-injection QD laser has indeed shown an enhanced small-signal modulation bandwidth and reduced temperature sensitivity of the threshold current density.

Figure 1 depicts a typical tunnel injection structure (TIS) - an additional QW is connected with a QD layer through a thin barrier and serves as a carrier reservoir/injector for the QDs layer [6]. Various InAs/InGaAs/InGaAlAs/InP material systems [7] have been already considered for TIS and even further modifications have been proposed [8].

The general microscopic mechanisms of carrier transfer in the dot-well structures are, however, far from being well understood [9]. In this paper we do not research all the aspects of the underlying physics of TIS, but concentrate only on the energy levels and their ordering as a function of the conduction band offset (CBO) between the host and the QD material. For lasers or amplifiers in the $1.55 \mu \mathrm{m}$ wavelength emission range the host can be made from $\operatorname{In}_{0.53} \mathrm{Ga}_{0.23} \mathrm{Al}_{0.24}$ As material lattice matched to $\mathrm{InP}$, an injector $\mathrm{QW}$ from $\mathrm{In}_{0.53} \mathrm{Ga}_{0.47} \mathrm{As}$ and $\mathrm{QDs}$ from InAs material, with possibly some admixture of Ga and $\mathrm{Al}$ due to intermixing during the growth. In the ideal

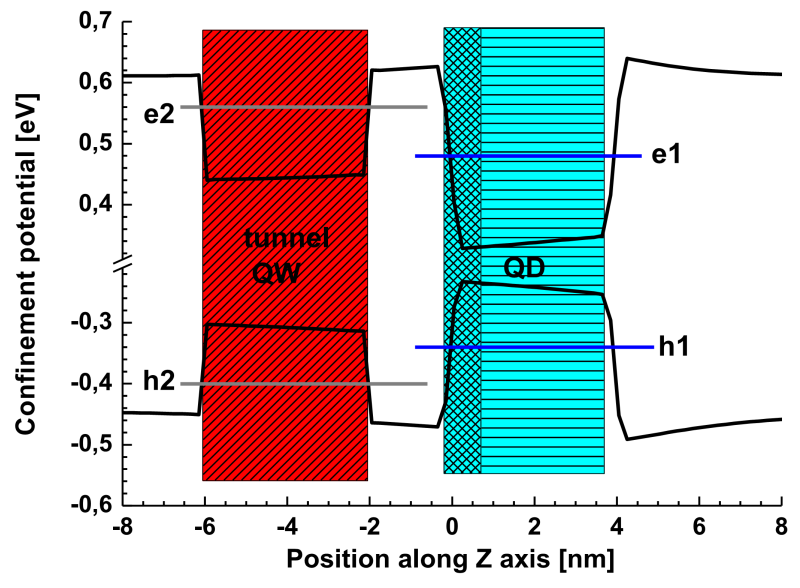

Fig. 1. Energy band profile for TIS structure. The positions of QW, WL and QD are marked with schematic desired energy levels positions in QD (e1 and h1) and QW (e2 and h2).

TIS, the lowest electron (e1) and the highest hole (h1) wave functions (WF) should remain in the QD, whereas the WFs of the first excited states for both electrons (e2) and holes (h2) should originate from the injector QW.

Moreover, the energy difference between the QW-like transition e2h2 and the QD-like transition e1h1 should be close to the lateral optical (LO) phonon energy for an efficient transfer of carriers.

Of course, there are many material parameters which influence energy levels with major or minor impact. Among such parameters, the CBO is one of these with the biggest impact on the electronic structure. In addition, the $\mathrm{CBO}$ has a clear and direct interpretation, but its value is hard to determine experimentally. Moreover, for a quaternary InGaAlAs material, its level of uncertainty is unacceptable if one wants to design and calculate an optimum tunnel QW-QD structure 


\section{Theoretical framework}

For the calculations, the own program based on a three-dimensional strain-dependent eight-band $\boldsymbol{k} \cdot \boldsymbol{p}$ model has been used $[10,11]$. It has several functionalities necessary for a proper calculation of this rather complicated quantum structure. The program is able to calculate the energy levels of a $\mathrm{QW}$ in a $3 \mathrm{D}$ geometric numerical box and it can simultaneously handle QDs of even large sizes, with different shapes. The computations are very efficient due to employing specially developed and original preconditioner for calculating the inner eigenvalues [11]. Basing on the strain calculated within the linear continuum mechanical elastic theory the nonlinear piezoelectric potential and the potential resulting from deformation potential are derived. Afterwards, the energy levels are calculated by using the multi-band approach developed by Bahder [12]. The realistic morphological parameters and compositional gradients are possible to be taken into account [13]. All physical parameters are taken after [14], except for the conduction band offset between the QD and the host material. A QD with planar sizes $12 \mathrm{~nm} \times 25 \mathrm{~nm}$ with $2.5 \%$ admixture of Ga and $\mathrm{Al}, 3 \mathrm{~nm}$ high is used, with a $2 \mathrm{~nm}$ barrier between the QD and the QW of different widths. The QDs are grown on a $0.9 \mathrm{~nm}$ thick wetting layer.

\section{Results and discussion}

In Fig. 2 the transition energies (solid lines with filled symbols) and their oscillator strengths (dashed lines with empty symbols) versus the conduction band offset for QW widths of $4 \mathrm{~nm}$ (a) and $8 \mathrm{~nm}$ (b) are shown (a very similar situation takes place for a QW $6 \mathrm{~nm}$ and $10 \mathrm{~nm}$ wide). At low values of the CBO (i.e., small confinement for electrons in the QD) electrons are mostly in the QW area, whereas holes are in the QD. Therefore, the oscillator strength is small. When the CBO increases, the electrons shift into the QD and so the oscillator strength increases. The total transition energy goes up because the energy of the hole level (which is QD-like) decreases with the increasing $\mathrm{CBO}$, while the electron energy level remains almost unaffected. For the $\mathrm{CBO}$ in the range about $50-60 \%$, both e1 and e2 electrons are in the QW area and their energy levels remain unchanged with the increasing CBO. Therefore, the parallel behaviour of the e1h1 and e2h1 transitions may be observed.

With further increase of CBO the first electron WF becomes more and more QD-like - which increases the oscillator strength of the related optical transition. For the $\mathrm{CBO}$ in the range of $75-80 \%$, two qualitatively different situations occur. In the wider QW, the first hole WF becomes QW-like. Thus the oscillator strength for the $\mathrm{e} 2 \mathrm{~h} 1$ transition (within $\mathrm{QW}$ region) is high. The opposite situation takes place for a narrower $\mathrm{QW}$ layer the difference being in the behaviour of the second electron energy level. Its WF remains confined in the QD, whereas for the wider QW the second electron $\mathrm{WF}$ is within QW. For the CBO larger than $80 \%$ both electron WFs are positioned in the QD.

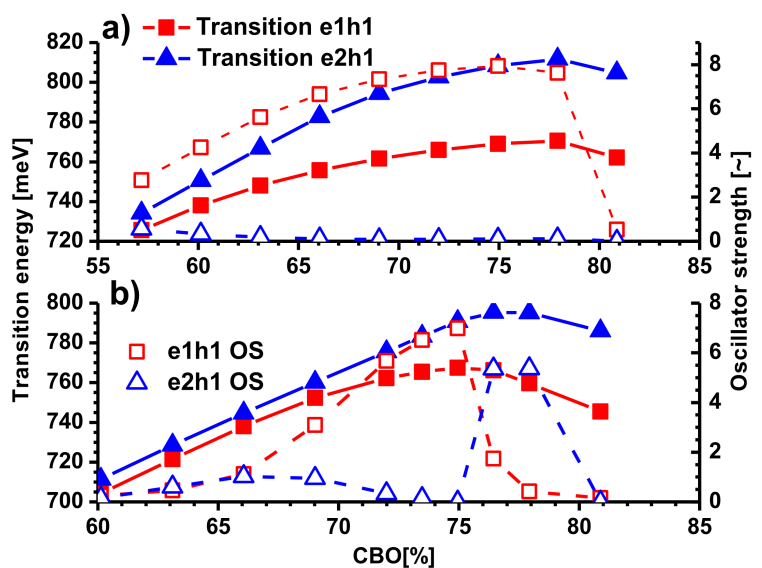

Fig. 2. Transition energies (solid lines and filled symbols) and oscillator strengths (OS) (dashed lines and empty symbols) for various CBO of the QD materials for TIS with $4 \mathrm{~nm}$ (a) and $8 \mathrm{~nm}$ (b) wide QWs. The rectangles (filled or empty) are used for e1h1 transitions, the triangles (filled or empty) are for e2h1 transitions.

In the case of the $8 \mathrm{~nm}$ and $10 \mathrm{~nm}$ wide QWs the situation may be ideal, i.e., both the lowest conduction band state and the highest valence band state of the entire system are QD-like, and the next higher energy conduction and valence band states are QW-like. Here, the energy difference between the QD-like transition and the QW-like one is $23 \mathrm{meV}$ for $\mathrm{CBO}=70 \%$ and $18 \mathrm{meV}$ for $\mathrm{CBO}=74 \%$ for the narrower $\mathrm{QW}$, and for the wider QW the energy difference is $14 \mathrm{meV}$ for $\mathrm{CBO}=70 \%$ and $10 \mathrm{meV}$ for $\mathrm{CBO}=74 \%$.

For a thin QW (i.e., $4 \mathrm{~nm}$ and $6 \mathrm{~nm}$ wide), only electrons may be injected from the QW to the QD ground state, since the first and second hole levels are QD-like. However, the injection of holes is less important, as they generally relax faster in the QD (the distance between consecutive hole levels is small) or they may be provided by $p$ doping. The advantage for such QWs is that the wider range of the CBOs (65-75\%) is allowed.

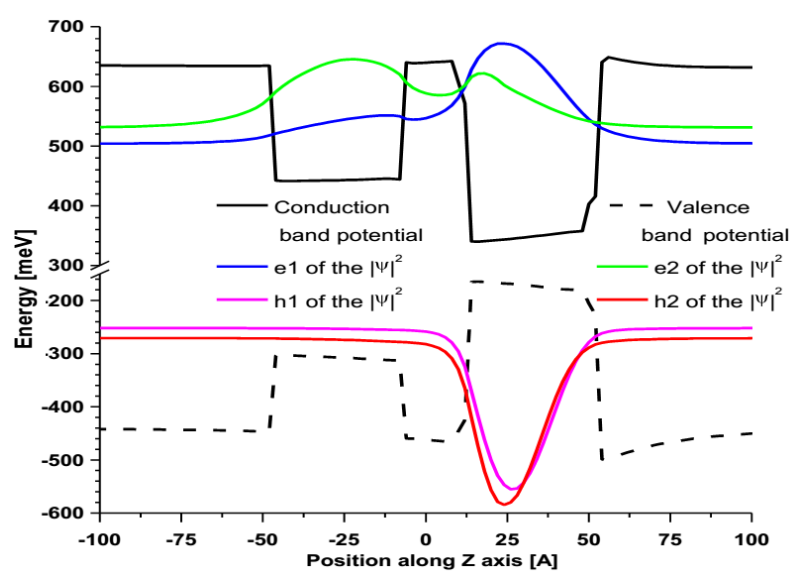

Fig. 3. Projection of the modulus squared of the wave functions $\left(|\psi|^{2}\right)$ along $Z$ axis for TIS for $\mathrm{CBO}=66 \%$ and $4 \mathrm{~nm}$ QW width. 


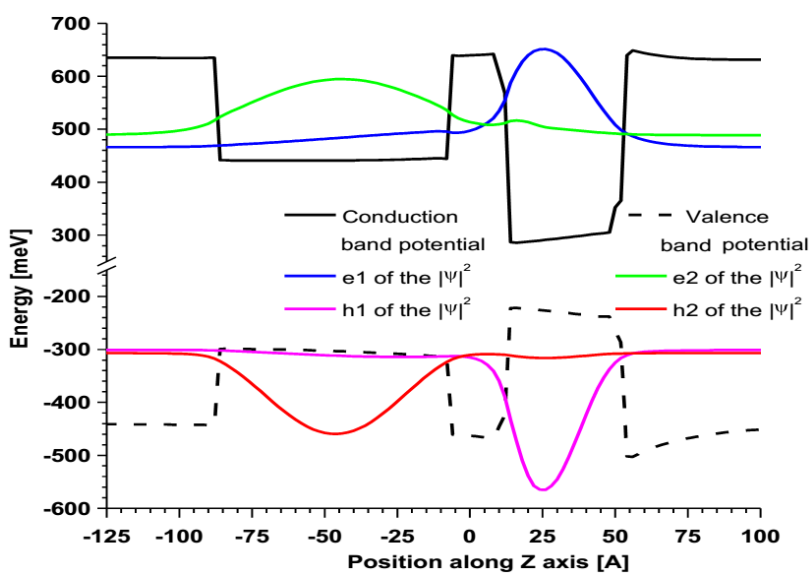

Fig. 4. Projection of the modulus squared of the wave functions $\left(|\psi|^{2}\right)$ along $Z$ axis for TIS for $\mathrm{CBO}=70 \%$ and $8 \mathrm{~nm}$ QW width.

In Figs. 3 and 4 there are shown the moduli squared of the WFs integrated over the plane perpendicular to the growth axis $Z$ (i.e., projection) versus the position along the $Z$ axis with $4 \mathrm{~nm}$ wide $\mathrm{QW}$ for $\mathrm{CBO}=66 \%$ and with $8 \mathrm{~nm}$ wide $\mathrm{QW}$, respectively. In the case of $4 \mathrm{~nm}$ wide QW, the e1 WF is mainly within QD but with noticeable part within QW. Similar situation could be observed for $\mathrm{e} 2 \mathrm{WF}$ - main part of the WFs are positioned in the tunnel QW but considerable fraction of WFs are also within QD. In Fig. 4 the situation is ideal - the $\mathrm{e} 1$ and $\mathrm{h} 1 \mathrm{WFs}$ are in the QD region with no penetration into QW. e2 and h2 WFs are exclusively within the injector QW.

Similar simulations performed for QDs with planar sizes $12 \mathrm{~nm} \times 75 \mathrm{~nm}$ and $12 \mathrm{~nm} \times 100 \mathrm{~nm}$ show that it is impossible to obtain the required energy levels ordering in the QD and QW. However, the QDs with large aspect ratios cannot be completely excluded, since the situation could be changed when the composition distribution within an individual nanostructure is taken into account [13].

\section{Conclusions}

In this work we have investigated the electronic structure of coupled $0 \mathrm{D}-2 \mathrm{D}$ structures - the influence of the $\mathrm{CBO}$ on the arrangement of the confined states in the QW and QD. It occurs that the knowledge on this difficulty to determine experimentally parameter is essential for the correct ordering of energy levels and whether the WFs are inside or outside the QD. The estimated optimum value of the CBO parameter would be in the range of $65-75 \%$, and it is essential to point out that it is less than the commonly used value [14] - 77\%. Therefore, the CBO needs reliable experimental verification before such structures are fabricated or used in the lasers. We have also shown that it is possible to design an almost ideal TIS structure where both carriers could be injected from the QW to the QD layer. In the particular case - for the $8 \mathrm{~nm}$ wide QW, with the CBO equal to $70 \%$, the difference between the QD-like and the
QW-like transitions is of the order of energy of the LO phonon energy. For $4 \mathrm{~nm}$ and $6 \mathrm{~nm}$ tunnel QW widths, only electrons could be injected to QD. However, it is not possible to obtain the TIS for the QDs with planar sizes of $12 \mathrm{~nm} \times 100 \mathrm{~nm}$ or $12 \mathrm{~nm} \times 75 \mathrm{~nm}$ - at least when one assumes no interdiffusion of $\mathrm{Ga}$ and $\mathrm{Al}$ from the barrier.

\section{Acknowledgments}

This work has been conducted within the Harmonia Project, National Science Centre of Poland, grant No. 2013/10/M/ST3/00636. I would like to thank Wojciech Rudno-Rudziński, Marcin Syperek and Grzegorz Sęk for fruitful discussions and critical reading of the manuscript.

\section{References}

[1] Y. Arakawa, H. Sakaki, Appl. Phys. Lett. 40, 939 (1982).

[2] L.V. Asryan, R.A. Suris, IEEE J. Quantum Electron. 34, 841 (1998).

[3] L.V. Asryan, R.A. Suris, S. Luryi, IEEE J. Quantum Electron. 39, 404 (2003).

[4] K. Kamath, D. Klotzkin, P. Bhattacharya, Proc. IEEE LEOS 10th Annual Meeting 2, 498 ( 1997).

[5] T. Chung, G. Walter, N. Holonyak, Jr., Appl. Phys. Lett.79, 4500 (2001).

[6] P. Bhattacharya, S. Ghosh, S. Pradhan, J. Singh, Z.K. Wu, J. Urayama, K. Kim, T.B. Norris, IEEE J. Quantum Electron. 39, 952 (2003).

[7] G. Sęk, R. Kudrawiec, P. Podemski, J. Misiewicz, A. Somers, S. Höfling, J.P. Reithmaier, M. Kamp, A. Forchel, J. Appl. Phys. 112, 033520 (2012).

[8] D.-S. Han, L.V. Asryan, Nanotechnology 21, 015201 (2010) and references therein.

[9] Ch. Wang, F. Grillot, J. Even, IEEE J. Quantum Electron. 48, 1144 (2012).

[10] J. Andrzejewski, G. Sęk, E. O'Reilly, A. Fiore, J. Misiewicz, J. Appl. Phys. 107, 073509 (2010).

[11] J. Andrzejewski, J. Comput. Phys. 249, 22 (2013).

[12] T.B. Bahder, Phys. Rev. B 41, 11992 (1990).

[13] A. Maryński, G. Sęk, A. Musiał, J. Andrzejewski, J. Misiewicz, C. Gilfert, J.P. Reithmaier, A. Capua, O. Karni, D. Gready, G. Eisenstein, G. Atiya, W.D. Kaplan, S. Kölling, J. Appl. Phys. 114, 094306 (2013).

[14] I. Vurgaftman, J.R. Meyer, L.R. Ram-Mohan, J. Appl. Phys. 89, 5815 (2001). 